\title{
Combined Treadmill Running and Insulin Therapy Favors the Stabilization of Glycemic Metabolic Parameters and Avoids Increased Achilles Tendon Rigidity in Diabetic Rats
}

\author{
A. C. Nobre de Lacerda Brito ${ }^{1}$, W. Alves Martins' ${ }^{1}$ P. C. da Silva Queiroz ${ }^{1}$, S. E. \\ Veríssimo Santos ${ }^{1}$, T. Meireles Santos ${ }^{2}$, R. F. de Souza ${ }^{3,4}$, D. Dutra Pereira ${ }^{3}$, M. T. \\ Gonzaga Santos ${ }^{4}$, A. C. Falcão Esteves ${ }^{1}$, S. R. Arruda de Moraes ${ }^{1}$ \\ 1 Laboratory of Neuromuscular Plasticity, Department of Anatomy, Center of Biosciences, Federal University of \\ Pernambuco, Recife, Brazil \\ 2 Department of Physical Education, Federal University of Pernambuco, Recife, Brazil \\ 3 Department of Physiology and Pharmacology, Center of Biosciences, Federal University of Pernambuco, Recife, \\ Brazil \\ ${ }^{4}$ Department of Physical Education, Federal University of Sergipe - UFS, São Cristovão, Sergipe, Brazil
}

\section{CORRESPONDING AUTHOR:}

Ana Camila Nobre de Lacerda Brito

Federal University of Pernambuco

Neuromuscular Plasticity Laboratory

Department of Anatomy

Avenida Professor Moraes Rego, 1235,

Recife 50670-901

Pernambuco, Brazil

E-mail: britoanacamila@gmail.com

DOI:

10.32098/mltj.03.2020.14

LEVEL OF EVIDENCE: 2B

\section{SUMMARY}

Background. Hyperglycemia reduces tendon homeostasis. Effects of physical exercise on diabetic rats have been widely studied; however, the effects of a combined physical and insulin therapy on biomechanical properties of the Achilles tendon (AT) remain unclear. Therefore, the objective of this study was to evaluate the effects of the combination of moderate-intensity exercise on a treadmill and insulin therapy on metabolism, physical conditioning, and biomechanics of AT in diabetic rats.

Methods. Forty-eight Wistar rats were divided into six groups: Sedentary ControlSCG, Treadmill Control-TCG, Sedentary Diabetic-SDG, Sedentary Insulin Diabetic-SIDG, Treadmill Diabetic-TDG, and Treadmill Insulin Diabetic-TIDG. Diabetic animals were induced with streptozotocin diluted in sodium citrate buffer $(50 \mathrm{mg} / \mathrm{Kg}$; $10 \mathrm{mM}$; pH 4.5; intraperitoneally). All groups were subjected to the maximal effort test for pre $\left(\mathrm{MET}_{1}\right)$ and post $\left(\mathrm{MET}_{2}\right)$ maximal speed determination. The exercise protocol was administered for 5 weeks ( $1 \mathrm{~h} /$ day, 5 days/week). Blood glucose levels and biomechanical properties of AT (e.g., traction) were evaluated.

Results. Increased glycemia was observed in SDG $(\mathrm{p}<0.001 ; \mathrm{p}=0.003)$, SIDG $(\mathrm{p}=0.009 ; \mathrm{p}=0.037)$, and TDG $(\mathrm{p}=0.002 ; \mathrm{p}=0.009)$; however, compared with SCG and TCG, TIDG showed no significant differences. Maximal force was reduced in TIDG $(\mathrm{p}=0.009)$ and SIDG $(\mathrm{p}=0.002)$ and increased in SCG and TDG compared with in SIDG $(\mathrm{p}=0.024)$. The elastic modulus was reduced in TCG compared with in SCG $(\mathrm{p}=0.011)$ and increased in SDG $(\mathrm{p}<0.001)$, SIDG $(\mathrm{p}=0.019)$, and TDG $(\mathrm{p}=0.006)$ compared with in TCG.

Conclusions. The combined physical exercise and insulin therapy favors the stabilization of glycemic metabolic parameters and avoids increased tendon rigidity in diabetic rats.

\section{KEY WORDS}

Achilles tendon; diabetes mellitus; maximal effort test. 


\section{BACKGROUND}

Regular physical exercise concomitant with hyperglycemic control has been widely investigated for the treatment of type 1 diabetes mellitus (DM1) $(1,2)$. Therefore, insulin therapy (IST) is commonly used to control hyperglycemia and other complications of diabetes, such as diabetic ketoacidosis and hyperosmolar states (2).

Insulin therapy is indicated for DM1 cases (3) because high glycemic indexes are associated with cardiometabolic (4) and musculoskeletal disorders (4-7), including tendon damage such as diabetic tendinopathy $(5,8)$.

Evidence suggests that hyperglycemia reduces tendon homeostasis as a consequence of a series of changes, including vascular hyperplasia, disorganized collagen fibers, decrease collagen production, increased advanced glycation end products (AGEs) (9-11), and altered viscoelastic properties (12). Such changes may increase tendon stiffness, thereby inducing diabetic tendinopathy (8) and causing degeneration and rupture (5).

Meanwhile, when physical exercise was regularly performed, physiological and biomechanical benefits, such as increased collagen concentration, fibrillary density (13), elastic module, maximal tension, cross-sectional energy/ area, and maximal force (14) as well as decreased thickness and neovascularization (15), were observed in the Achilles tendon (AT) of patients with calcaneus tendinopathy $(15,16)$.

Recent studies in DM1-induced animals have reported positive effects of moderate-intensity aerobic exercise (running and swimming) on the restoration of the elastic characteristics of AT, preventing the progression of tendon degeneration $(17,18)$. However, these studies did not implement IST during exercise. Assuming that IST can prevent the onset of musculoskeletal chronic complications (18) and optimize motor skills in DM1-induced rats (19), we hypothesized that IST concomitant with physical exercise can boost the biomechanical properties of AT.

Considering that some precautions should be ensured regarding exercise practice in patients with diabetes to appropriately prescribe safe exercise intensity, in the present study, a moderate-intensity treadmill exercise standardization model was adopted using the maximal effort test proposed by Brito et al. (20).

The present study aimed to investigate the effects of a combination of moderate-intensity aerobic exercise on a treadmill and IST on the biomechanical properties of AT in as well as the clinical status and physical fitness of DM1-induced rats.

\section{MATERIALS AND METHODS}

\section{Experimental design}

To evaluate the effects of the combination of IST and moderate-intensity treadmill exercise on AT in healthy and diabetic rats, this study was divided into four stages: (1) distribution of animals into six groups, induction of experimental diabetes, and onset of IST; (2) adaptation to the treadmill and administration of the first maximal effort test $\left(\mathrm{MET}_{1}\right)$; administration of the 5 -week protocol of moderate-intensity treadmill exercise and the second maximal effort test $\left(\mathrm{MET}_{2}\right)$; material collection and administration of AT traction biomechanical test. The study was performed at the Neuromuscular Plasticity Laboratory of the Anatomical Department and the Biopolymer Laboratory of the Chemical Engineering Department of the Federal University of Pernambuco (UFPE).

Forty-eight male Wistar rats $(234.4 \pm 28.1 \mathrm{~g})$ were housed in cages at $23 \pm 1^{\circ} \mathrm{C}$ under a 12 -h inverted light/dark cycle. Presence Food (Neovia, São Paulo, Brazil) and water were provided ad libitum. Animals that did not present a fasting blood glucose level $>200 \mathrm{mg} \cdot \mathrm{dL}^{-1}$ on day 7 after the induction of experimental diabetes, did not continuously run during the adaptation week, and reached a $\mathrm{MET}_{1}$ maximal speed $<10 \mathrm{~m} \cdot \mathrm{min}^{-1}$ were excluded. Ethical approval of the study was obtained from the Committee of the Federal University of Pernambuco Bioscience Center (number 23076.050209/2016-10) in accordance with the norms recommended by the Brazilian Committee for Animal Experimentation. The research was conducted ethically according to international standards and as required by the journal (21).

\section{PROCEDURES}

\section{Animal distribution into groups and induction of experimental diabetes}

At the age of 50 days, animals were randomly assigned to six groups ( $\mathrm{n}=8$ per group): sedentary control group (SCG), treadmill control group (TCG), sedentary diabetic group (SDG), treadmill diabetic group (TDG), sedentary insulin diabetic group (SIDG), and treadmill insulin diabetic group (TIDG). Experimental diabetes was induced in animals in the diabetic groups at the age of 60 days, and after $12 \mathrm{~h}$ of fasting, measure body weight (FILIZOLA BP6 Digital Scale) and blood glucose (Accu-Chek Active Kit Glucometer) were measured. Experimental diabetes was induced 
via the administration of streptozotocin $\left(50 \mathrm{mg} \cdot \mathrm{kg}^{-1}\right.$; STZ, Sigma Aldrich Co., St. Louis, MO) diluted in sodium citrate buffer (10 mM; pH 4.5; intraperitoneally [i.p.])(22). Animals in the control groups received an injection of sodium citrate buffer alone (23).

\section{Insulin therapy}

From day eight after the induction of experimental diabetes, insulin (Eli Lilly and Company) was subcutaneously administered (24) (2 IU/day/mouse; HUMULIN ${ }^{\circledR} \mathrm{N}$ ) daily to animals in SIDG and TIDG, 4-6 h after the exercise sessions and throughout the experimental protocol (3).

\section{Treadmill adjustment period, MET, and moderate-intensity treadmill exercise protocol}

All animals were subjected to treadmill adaptation nine days after the induction of experimental diabetes. Treadmill adaptation was performed for three days $\left(10\right.$ min.day $^{-1}$; $5 \mathrm{~m} \cdot \mathrm{min}^{-1} ; 0^{\circ}$ inclination) on the multiple rodent treadmill (AVS Projects). On day 4, MET 1 was administered at an initial speed of $5 \mathrm{~m} \cdot \mathrm{min}^{-1}$, with a progressive increase in speed by $5 \mathrm{~m} \cdot \mathrm{min}^{-1}$ every $3 \mathrm{~min}$. The maximum intensity for each rat was determined when the animal touched the back wall of the treadmill belt five times within 1 min $(19,20,22)$. After $72 \mathrm{~h}$ of $\mathrm{MET}_{1}$, the adapted protocol (19) was initiated at moderate intensity $(20,25)$ in animals in TCG, TDG, and TIDG over 5 weeks ( $0^{\circ}$ inclination; $1 \mathrm{~h} /$ day; 5 days/week). Each session comprised three periods: warm-up, moder- ate-intensity exercise, and recovery. During the first week, time and speed were progressively increased. To encourage the animals, a low-intensity electrical stimulus (1.5-2.0 mA) located at the end of each lane was used.

Approximately $48 \mathrm{~h}$ after the last exercise session, $\mathrm{MET}_{2}$ was administered. All protocols are detailed in table $\mathbf{I}$.

\section{Clinical and metabolic evaluation, material collection, and euthanasia}

After the experimental period, weight and blood glucose levels were measured in all animals. This was followed by anesthesia with xylazine solution and ketamine hydrochloride (Anasedan ${ }^{\circledR}, 20 \mathrm{mg} \cdot \mathrm{kg}^{-1}$ and Dopalen ${ }^{\circledR}, 100 \mathrm{mg} \cdot \mathrm{kg}^{-1}$, respectively; $0.1 \mathrm{~mL}$ and $0.1 \mathrm{~mL}$ for each $100 \mathrm{~g}$ of animal weight, respectively, intraperitoneal). An incision was made on the upper surface of the right paw for the visualization and dissection of the myotendinous complex of the triceps surae muscle while preserving the distal fixation of the calcaneal bone and the animal's paw. The anatomical sample was moistened with saline and sent to the Biopolymer Laboratory at UFPE for biomechanical assay. After the collection of biological material and while still under the effect of anesthesia, animals were euthanized via the intracardiac injection of $1 \mathrm{~mL}$ potassium chloride $(10 \%)$.

\section{Tendon traction mechanical test}

Biomechanical testing was performed on the mechanical tensile testing conventional machine (EMIC, Model DL 500,

Table I. Treadmill exercise protocol applied in the diabetic treadmill group (TDG) and diabetic treadmill insulin group (TIDG).

\begin{tabular}{|c|c|c|c|}
\hline Protocol & Week or Day & Time (minutes) & Training \\
\hline \multirow[b]{2}{*}{ Adaptation } & 1st day & 10 & \multirow[b]{2}{*}{ Velocity: $5 \mathrm{~m} \cdot \mathrm{min}^{-1}$} \\
\hline & 2nd day & 10 & \\
\hline Maximum effort test & 4th day & Varies according to animal & $\begin{array}{l}\text { Initial velocity of } 5 \mathrm{~m} \cdot \mathrm{min}^{-1} \text { being increased every } 3 \mathrm{~min} \text { at } \\
\qquad 5 \mathrm{~m} \cdot \mathrm{min}^{-1} \text { until fatigue }\end{array}$ \\
\hline \multirow{4}{*}{$\begin{array}{l}\text { Moderate intensity } \\
\text { exercise }\end{array}$} & 3rd day & 40 & $5 \mathrm{~min}$ at $30 \%_{\mathrm{MET1}}+30 \mathrm{~min}$ at $50 \%_{\mathrm{MET1}}+5 \mathrm{~min}$ at $30 \%_{\mathrm{METT}}$ \\
\hline & 4th day & 50 & $5 \mathrm{~min}$ at $30 \%_{\mathrm{MET} 1}+40 \mathrm{~min}$ at $50 \%_{\mathrm{MET1}}+5 \mathrm{~min}$ at $30 \%_{\mathrm{MET1}}$ \\
\hline & 5 th day & 55 & $5 \mathrm{~min}$ at $30 \%_{\mathrm{MET1}}+45 \mathrm{~min}$ at $50 \%_{\mathrm{MET1}}+5 \mathrm{~min}$ at $30 \%_{\mathrm{MET} 1}$ \\
\hline & 2nd week & 60 & $5 \mathrm{~min}$ at $30 \%_{\mathrm{MET1}}+50 \mathrm{~min}$ at $60 \%_{\mathrm{MET} 1}+5 \mathrm{~min}$ at $30 \%_{\mathrm{MET} 1}$ \\
\hline
\end{tabular}

Min - minutes; \% - percentage; $\mathrm{MET}_{1}$ : Maximum Speed Test. 
Brazil, $500 \mathrm{~N}$ ). The ellipse formula was applied to measure the tendon cross-sectional area (26), as shown in Equation I. Subsequently, the sample was connected to the machine via two pieces that were previously made for this type of biological material, keeping the sample in the anatomical position. The length of the sample was then measured using a caliper (Vernier Calipers, $0.05 \mathrm{~mm}$ ), and specimen traction was performed at a speed of $0.1 \mathrm{~mm} \cdot \mathrm{s}^{-1}$ to its point of failure. Maximal force, maximal force tension, cross-sectional energy/area, maximal strain, specific strain, and elastic modulus were calculated using the stress vs. strain graph generated by the Tesc ${ }^{\circledR}$ software coupled with the biomechanical testing machine.

$$
\operatorname{CSA}=(D \times d / 4) \times \pi
$$

\section{Where:}

CSA = the cross-sectional area;

$\mathrm{D}=$ the larger longest of the medial region of AT;

$\mathrm{d}=$ the shortest diameter of the medial region of AT;

and $\pi=3.1416$

\section{STATISTICAL ANALYSIS}

After verifying the data assumptions for parametric analysis using Kolmogorov-Smirnov test, one-way ANOVA was performed, followed by post hoc Bonferroni test, for the analysis of variables with normal distribution. When normality was not verified, Kruskal-Wallis test was used, followed by pairwise comparison. Parametric data were presented as mean and standard deviation and non-parametric data as median and interquartile range. To assess the effect of size, Cohen's $d$ (27) was used. All analyses were performed using SPSS version 20. A significance level of 5\% was adopted.

\section{RESULTS}

Of the 32 animals with induced diabetes, two did not exhibit a blood glucose level $>200 \mathrm{mg} . \mathrm{dL}^{-1}$ on day 7 after the induction of experimental diabetes and were excluded from the study. At the beginning of the study, all animals presented homogeneous parameters of body weight $(p=0.367)$ and glycemia $(p=0.094)$. At the end of the exercise protocol, blood glucose levels and body weights in TIDG were similar to those in SCG $(\mathrm{p}=1.000)$ and TCG $(\mathrm{p}=1.000)$. However, compared with the nondiabetic groups (SCG and TCG), the diabetic groups (SDG, SIDG, and TDG) exhibited increased glycemic index $(\mathrm{p}<0.05)$. Reduction in body weight was observed only in SDG (SDG vs. SCG: $\downarrow 24.03 \%$, $\mathrm{p}<0.001, d=2.34$ and SDG vs. TCG: $\downarrow 22.99 \%, \mathrm{p}=$ $0.003, d=2.41$ ). Conversely, compared with the nondiabetic groups, the diabetic groups with IST alone or combined with treadmill exercise showed similarity in body weight ( $\mathrm{p}$ $>0.05$; figure 1). Increased blood glucose was observed in
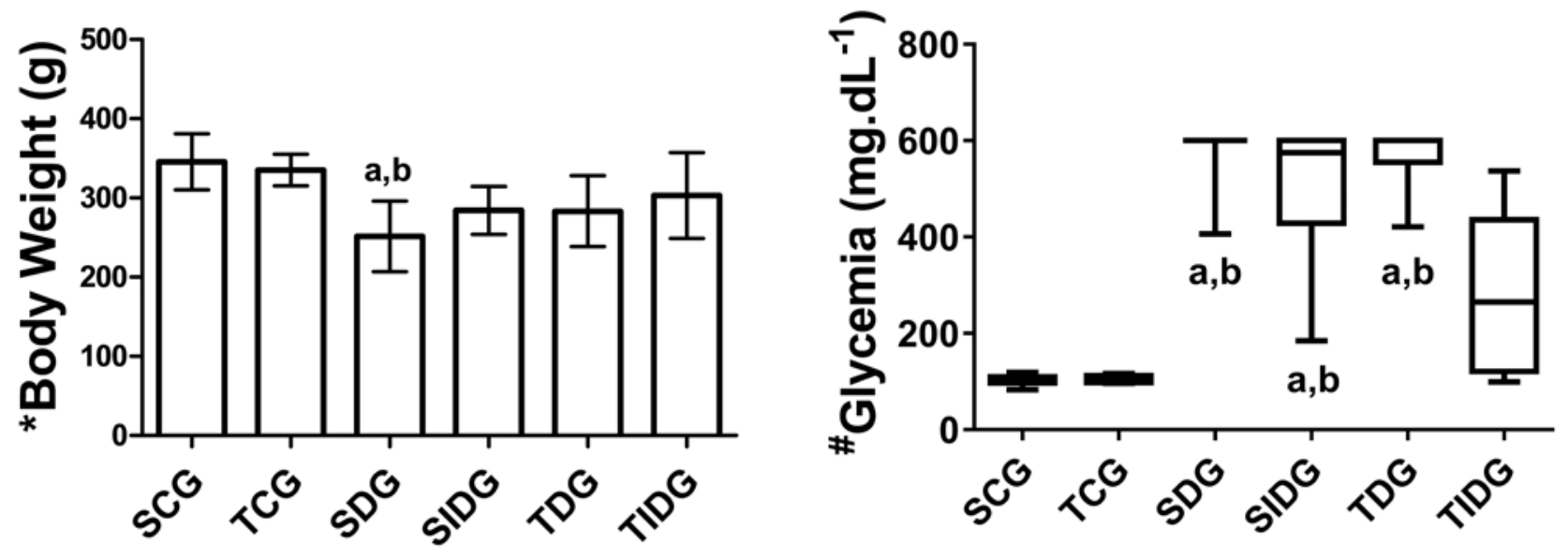

Figure 1. Body weight and glycemic indexes after five weeks of moderate treadmill exercise. SCG - Sedentary Control Group; TCG - Treadmill Control Group; SDG - Sedentary Diabetic Group; SIDG - Sedentary Insulin Diabetic Group; TDG - Treadmill Diabetic Group; TIDG - Treadmill Insulin Diabetic Group. Body weight values are expressed as mean \pm standard deviation. (*) Anova with post hoc of Bonferroni, whereas final glycemia values are expressed in median and interquartile range (\#) Kruskal Wallis with paired comparison to determine statistical difference between groups $(p<0.05)$. Where (a) represents the difference in relation to $S C G$, (b) difference in relation to TCG. 
all diabetic groups (SDG, SIDG, TDG, and TIDG) $(\mathrm{p}<$ 0.05) (figure 2).

In $\mathrm{MET}_{1}$, no differences in maximal speed were observed among the evaluated groups. In $\mathrm{MET}_{2}$, maximal speed was increased in TIDG compared with that in SDG $(\uparrow 17.03 \%$, $\mathrm{p}=0.002, d=2.97)$ and SIDG $(\uparrow 23.82 \%, \mathrm{p}=0.001, d=$
$2.35)$ and decreased in SDG $(\downarrow 11.11 \%, \mathrm{p}=0.020, d=3.88)$ and SIDG $(\downarrow 22.86 \%, \mathrm{p}=0.011, d=2.42)$ compared with that in TCG (figure 3).

Regarding the biomechanical properties of AT, maximal force was reduced in TIDG $(\downarrow 31.51 \%, \mathrm{p}=0.009, d=1.88)$ and SIDG $(\downarrow 32.23 \%, P=0.002, d=1.84)$ compared with
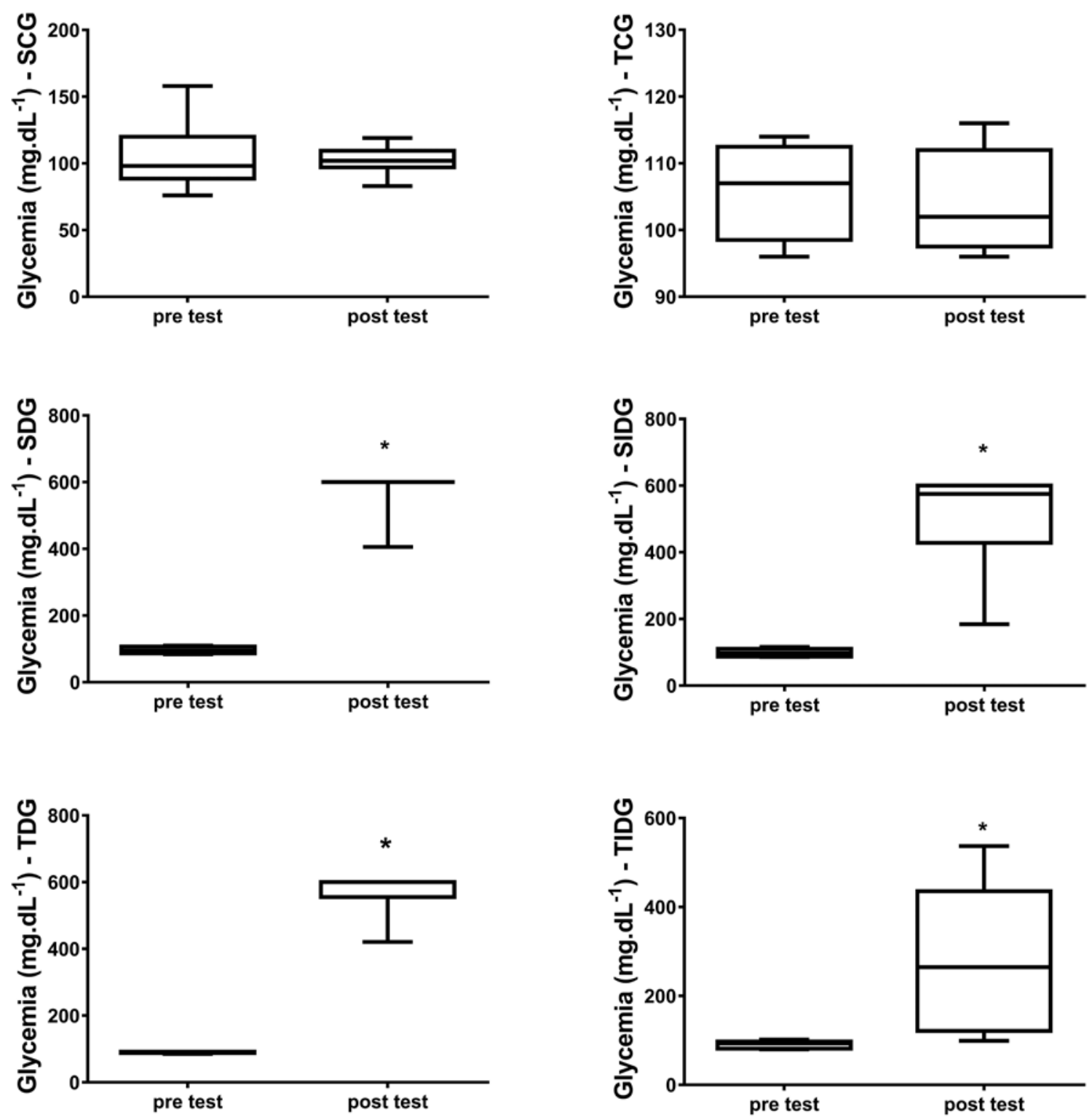

Figure 2. Evolution of Glycemia (mg.dL-1) before and after five weeks of moderate treadmill exercise in each group. SCG Sedentary Control Group; TCG - Treadmill Control Group; SDG - Sedentary Diabetic Group; SIDG - Sedentary Insulin Diabetic Group; TDG - Treadmill Diabetic Group; TIDG - Treadmill Insulin Diabetic Group. Body weight values are expressed as median and interquartile range. Wilcoxon test $(p<0.05)$. 


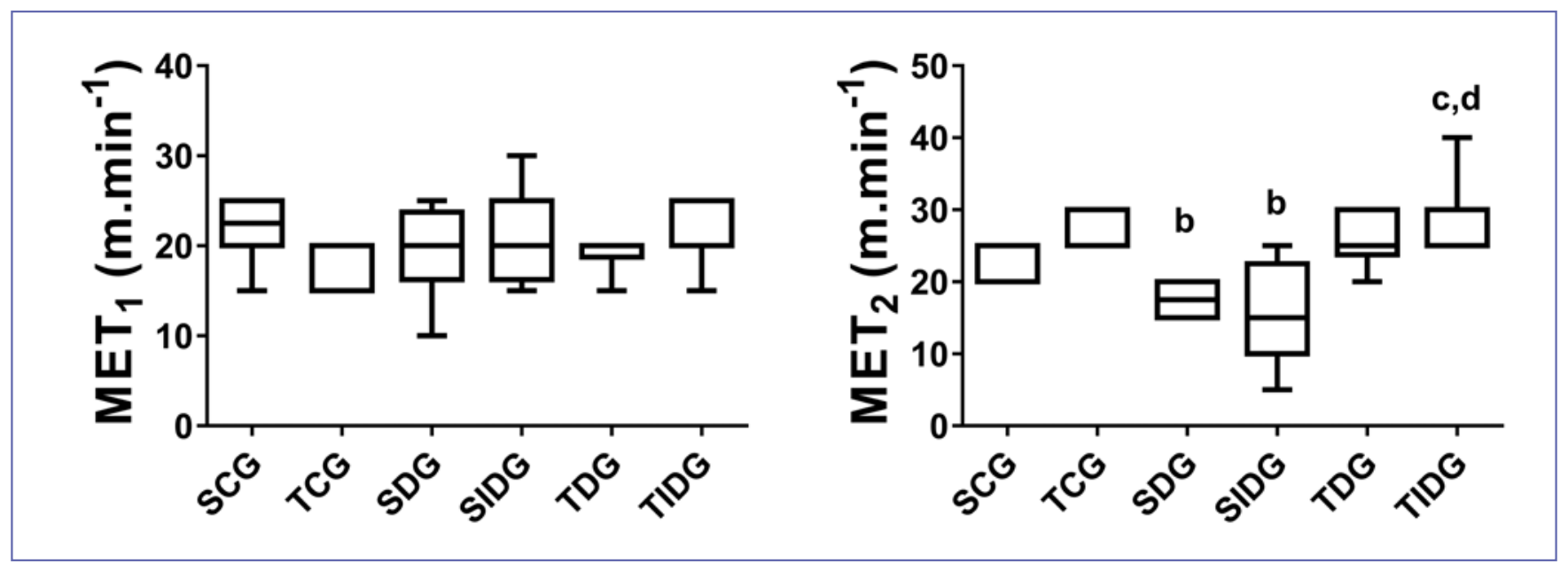

Figure 3. Maximum speed values $\left(\mathrm{m} \cdot \mathrm{min}^{-1}\right)$ achieved by the animals in the maximal stress tests before and after five weeks of moderate treadmill Exercise. MET1 - Maximum speed reached by animals in the maximum exercise test performed before the exercise protocol; MET2 - Maximum speed reached by the animals in the maximum exercise test performed after the exercise protocol; SCG - Sedentary Control Group; TCG - Treadmill Control Group; SDG - Sedentary Diabetic Group; SIDG - Sedentary Insulin Diabetic Group; TDG - Treadmill Diabetic Group; TIDG - Treadmill Insulin Diabetic Group. Values are expressed as median and interquartile range. Kruskal Wallis $(p<0.05)$ was used with paired comparison to determine statistical difference between groups. Where, (b) difference from TCG, (c) difference from SDG, (d) difference from SIDG.

that in SCG and increased in TDG compared with that in SIDG $(\uparrow 47.78 \%, \mathrm{p}=0.024, d=1.40)$. Moreover, elastic modulus was decreased in TCG compared with that in SCG $(\downarrow 37.79 \%, \mathrm{p}=0.011, d=1.59)$ and increased in SDG $(\uparrow 28.32 \%, \mathrm{p}<0.001, d=2.06)$, SIDG $(\uparrow 24.22 \%, \mathrm{p}=$ $0.019, d=2.32)$, and TDG ( $\uparrow 53.23 \%, \mathrm{p}=0.006, d=1.06)$ compared with that in TCG. There was no difference in the other biomechanical parameters of AT among the evaluated groups (table II).

\section{DISCUSSION}

In this study, we aimed to investigate the biomechanical properties of AT as well as the clinical status and physical fitness of DM1-induced rats following a combination of IST and moderate-intensity aerobic exercise on a treadmill, with the exercise speed previously verified through the maximum effort test, thus ensuring the biological individuality and taking into account the pathological state of these animals. Given this, IST combined with five weeks of exercise prevented the increase in the elastic modulus of AT, favored glycemic balance, and increased physical fitness.

Similarly, the analysis of biomechanical and structural properties of AT indicated that IST without physical exercise may decrease maximal strength, and sedentary rats exhibited increased elastic modulus. Collectively, these results suggest an adaptive exercise response, which is optimized by IST; this is in accordance with the results of recent studies $(3,28)$, which reported that regardless of IST being potentially relevant to the health of individuals with DM1, complementary associations and other therapies are necessary to counteract the triggered chronic complications.

Physical exercise aids glycemic control because it increases insulin-receptor sensitivity and facilitates glycolytic removal (29). Although the practice of moderate and regular physical activity alone is beneficial for DM1, we found that in combination with IST, it favored glycemic control, which was not observed in untrained diabetic animals. This implies that the induction of experimental diabetes results in hyperglycemia.

Our findings corroborate that even without the introduction of a low-carbohydrate diet, which is part of the adequate dietary planning for DM1 treatment (1), the combination of moderate-intensity aerobic exercise on a treadmill with IST benefits metabolic balance, possibly because of glycogenolysis and muscle energy production from physical exercise (30). Future studies should be conducted considering the results presented herein association with diet manipulation to test the hypothesis of benefits potentization.

Insulin combined with moderate-intensity exercise on a treadmill increased the physical fitness of DM1 animals, whereas sedentary animals exhibited decreased physical fitness regardless of IST use. Physical inactivity combined with a hyperglycemic state potentiates the chronic compli- 
Table II. Biomechanical and structural parameters of the Achilles tendon in Wistar rats after five weeks of treadmill exercise with moderate intensity.

\begin{tabular}{|c|c|c|c|c|c|c|c|}
\hline Parameters & $\begin{array}{l}\text { SCG } \\
(n=8)\end{array}$ & $\begin{array}{l}\text { TCG } \\
(n=5)\end{array}$ & $\begin{array}{l}\text { SDG } \\
(n=8)\end{array}$ & $\begin{array}{l}\text { SIDG } \\
(n=8)\end{array}$ & $\begin{array}{l}\text { TDG } \\
(n=6)\end{array}$ & $\begin{array}{l}\text { TIDG } \\
(n=8)\end{array}$ & $\begin{array}{c}\text { Kruskal- } \\
\text { Wallis }\end{array}$ \\
\hline $\begin{array}{l}\text { Cross-sectional } \\
\text { Area } \\
(\mathrm{mm} 2)\end{array}$ & $\begin{array}{c}1,26 \\
(1,12-1,48)\end{array}$ & $\begin{array}{c}1,25 \\
(1,10-1,71)\end{array}$ & $\begin{array}{c}0,93 \\
(0,81-1,07)\end{array}$ & $\begin{array}{c}1,10 \\
(1,03-1,30)\end{array}$ & $\begin{array}{c}1,28 \\
(0,87-1,51)\end{array}$ & $\begin{array}{c}1,06 \\
(0,91-1,19)\end{array}$ & 0,074 \\
\hline $\begin{array}{l}\text { Length of tendon } \\
(\mathrm{mm})\end{array}$ & $\begin{array}{c}2,39 \\
(1,84-3,21)\end{array}$ & $\begin{array}{c}1,78 \\
(1,48-2,21)\end{array}$ & $\begin{array}{c}2,43 \\
(1,86-2,77)\end{array}$ & $\begin{array}{c}2,40 \\
(1,75-2,93)\end{array}$ & $\begin{array}{c}2,24 \\
(1,96-2,99)\end{array}$ & $\begin{array}{c}1,63 \\
(1,37-2,38)\end{array}$ & 0,187 \\
\hline $\begin{array}{l}\text { Maximum Tension } \\
(\mathrm{MPa})\end{array}$ & $\begin{array}{c}25,69 \\
(23,17-29,60)\end{array}$ & $\begin{array}{c}24,59 \\
(15,47-28,58)\end{array}$ & $\begin{array}{c}32,36 \\
(26,08-38,06)\end{array}$ & $\begin{array}{c}23,02 \\
(19,22-25,41)\end{array}$ & $\begin{array}{c}24,89 \\
(22,19-35,86)\end{array}$ & $\begin{array}{c}25,61 \\
(22,95-28,46)\end{array}$ & 0,100 \\
\hline $\begin{array}{l}\text { Deformation } \\
\text { Maximum Strength } \\
(\mathrm{mm})\end{array}$ & $\begin{array}{c}2,69 \\
(2,18-3,19)\end{array}$ & $\begin{array}{c}2,87 \\
(2,69-3,39)\end{array}$ & $\begin{array}{c}2,61 \\
(2,03-3,04)\end{array}$ & $\begin{array}{c}2,43 \\
(1,94-2,80)\end{array}$ & $\begin{array}{c}2,33 \\
(1,73-2,77)\end{array}$ & $\begin{array}{c}2,10 \\
(1,84-2,64)\end{array}$ & 0,108 \\
\hline $\begin{array}{l}\text { Energy/Cross- } \\
\text { sectional Area } \\
(\text { N.mm/mm2) }\end{array}$ & $\begin{array}{c}48,65 \\
(31,26-56,26)\end{array}$ & $\begin{array}{c}37,97 \\
(24,98-50,52)\end{array}$ & $\begin{array}{c}49,58 \\
(35,45-58,58)\end{array}$ & $\begin{array}{c}31,05 \\
(28,30-42,73)\end{array}$ & $\begin{array}{c}31,55 \\
(26,67-56,77)\end{array}$ & $\begin{array}{c}33,65 \\
(28,86-39,79)\end{array}$ & 0,229 \\
\hline $\begin{array}{l}\text { Elastic Modulus } \\
\text { (MPa) }\end{array}$ & $\begin{array}{c}35,52 \\
(20,36-46,68)\end{array}$ & $\begin{array}{c}15,17 \\
(11,21-19,41) \mathbf{a}\end{array}$ & $\begin{array}{c}44,76 \\
(26,48-61,27) \mathbf{b}\end{array}$ & $\begin{array}{c}29,04 \\
(25,43-37,33) \mathbf{b}\end{array}$ & $\begin{array}{c}31,48 \\
(25,82-69,03) \mathbf{b}\end{array}$ & $\begin{array}{c}23,16 \\
(17,97-50,96)\end{array}$ & 0,018 \\
\hline
\end{tabular}

SCG - Sedentary Control Group; TCG - Treadmill Control Group; SDG - Sedentary Diabetic Group; SIDG - Sedentary Insulin Diabetic Group; TDG - Treadmill Diabetic Group; TIDG - Treadmill Insulin Diabetic Group. The values are median and and interquartile range. The Krukal-Wallis test was used, followed by the paired comparison to determine the statistical difference between the groups $(\mathrm{p}<0.05)$. Where (a) represents the difference in relation to SCG, (b) difference in relation to TCG.

cations of musculoskeletal $(5,31)$ and cardiovascular $(4,32)$ systems, which in turn can be mitigated by physical exercise $(33,34)$. Together, that evidence reinforces the clinical benefits of physical exercise for diabetes.

In AT, IST did not counteract the loss of maximal strength, both in sedentary and diabetic animals that exercised on the treadmill. However, this analysis did not consider the intrinsic characteristics of the biomaterial under study, specifically the cross-sectional area and length of AT. However, these properties determine the maximal tension, which was calculated by the ratio of the maximal force supported by the tendon relative to the cross-sectional area and elastic modulus, as quantified based on the maximal tension vs. specific deformation curve (18).

Although IST did not promote any change in maximal stress, the cross-sectional area, maximal and specific deformation, and elastic modulus were increased in the diabetic groups. However, this effect was not observed in groups that received IST combined with physical exercise. These findings corroborate the results of the study by Silva, Santos (31), which showed that sedentary diabetic animals that did not receive IST showed increased elastic modulus. Although this result can be interpreted as the facilitation of the transmission of muscle energy to the tendon in healthy rats, this increase is related to the disorganization of collagen fibers in diabetic animals, with tendons being more prone to rupture $(5,6)$.

In addition, increased elastic modulus is related to changes in the extracellular matrix components of the tendon because of prolonged hyperglycemia, with reduced proteoglycan levels and increased number of cross-links between collagen fibers being responsible for changes in the viscoelastic properties of the tendon (12,35). In turn, this decreases lubrication and increases slipping among collagen fibrils, consequently increasing tendon rigidity and promoting the development of diabetic tendinopathy (5). Furthermore, chronic hyperglycemia is associated with a significantly increased number of mast cells and vascular hyperplasia in the transverse area of blood vessels in AT as well as with the upregulation of the vascular endothelial growth factor, collagen type 1, inflammatory molecular indicators, such as nuclear factor kappa B (36), neutrophil 
infiltration, increased basophilia of the tenocytes, increased nuclear size/rounding (37).

Achilles tendon plays an important biomechanical role, particularly in gait and body stability. The mechanical changes observed in the diabetic groups in this study were characterized by changes in the properties of rodent AT, notably due to hyperglycemia and consequent fragility of this structure (37). However, this condition could be attenuated by regular aerobic exercise of moderate intensity combined with IST.

In summary, the combination of IST and moderate-intensity exercise performed on a treadmill in DMI1-induced rats enhanced the stability of glycemic metabolic parameters, thus avoiding an increase in tendon stiffness, as demonstrated by the elastic modulus values. Therefore,

\section{REFERENCES}

1. De Angelis K, da Pureza DY, Flores LJ, et al. [Physiological effects of exercise training in patients with type 1 diabetes]. Arquivos brasileiros de endocrinologia e metabologia. 2006;50(6):1005-13.

2. Diabetes SBd. Diretrizes da Sociedade Brasileira de Diabetes (2017-2018). Editora Clannad São Paulo; 2017.

3. Malarde L, Gratas-Delamarche A, Le Douairon-Lahaye S, et al. Endurance training and insulin therapy need to be associated to fully exert their respective beneficial effects on oxidant stress and glycemic regulation in diabetic rats. Free radical research. 2014;48(4):412-9.

4. Anaruma CP, Ferreira M, Jr., Sponton CH, Delbin MA, Zanesco A. Heart rate variability and plasma biomarkers in patients with type 1 diabetes mellitus: Effect of a bout of aerobic exercise. Diabetes research and clinical practice. 2016;111:19-27.

5. de Oliveira RR, de Lira KD, Silveira PV, Coutinho MP, et al. Mechanical properties of achilles tendon in rats induced to experimental diabetes. Annals of biomedical engineering. 2011;39(5):1528-34

6. Gautieri A, Redaelli A, Buehler MJ, Vesentini S. Age- and diabetes-related nonenzymatic crosslinks in collagen fibrils: candidate amino acids involved in Advanced Glycation End-products. Matrix biology : journal of the International Society for Matrix Biology. 2014;34:89-95.

7. Patrocínio-Silva TL, Souza AMFd, Goulart RL, Pegorari CF, et al. Low-level laser therapy associated to a resistance training protocol on bone tissue in diabetic rats. Archives of endocrinology and metabolism. 2016;60(5):457-64.

8. de Oliveira RR, Lemos A, de Castro Silveira PV, da Silva RJ, de Moraes SR. Alterations of tendons in patients with diabetes mellitus: a systematic review. Diabetic medicine : a journal of the British Diabetic Association. 2011;28(8):886-95.

9. de Oliveira RR, Martins CS, Rocha YR, et al. Experimental diabetes induces structural, inflammatory and vascular changes of Achilles tendons. PloS one. 2013;8(10):e74942.

10. Li Y, Fessel G, Georgiadis M, Snedeker JG. Advanced glycation end-products diminish tendon collagen fiber sliding. the present study supports the importance of combining these two therapies in preventing tendon injuries in diabetic patients. In addition, it suggests that new works be developed combining these therapies with adequate food planning.

\section{FUNDINGS}

This research was financed in part by the Cordenação de Aperfeiçoamento de Pessoal de Nivel Superior - Brazil (CAPES) - Finance Code 001.

\section{CONFLICTS OF INTERESTS}

The authors declare that they have no conflict of interests.
Matrix biology : journal of the International Society for Matrix Biology. 2013;32(3-4):169-77.

11. Mohsenifar Z, Feridoni MJ, Bayat M, Masteri Farahani R, Bayat S, Khoshvaghti A. Histological and biomechanical analysis of the effects of streptozotocin-induced type one diabetes mellitus on healing of tenotomised Achilles tendons in rats. Foot and ankle surgery : official journal of the European Society of Foot and Ankle Surgeons. 2014;20(3):186-91.

12. Burner T, Gohr C, Mitton-Fitzgerald E, Rosenthal AK. Hyperglycemia reduces proteoglycan levels in tendons. Connective tissue research. 2012;53(6):535-41.

13. Enwemeka CS, Maxwell LC, Fernandes G. Ultrastructural morphometry of matrical changes induced by exercise and food restriction in the rat calcaneal tendon. Tissue \& cell. 1992;24(4):499-510.

14. Bezerra MA, Santos de Lira KD, Coutinho MP, et al. Biomechanical and structural parameters of tendons in rats subjected to swimming exercise. International journal of sports medicine. 2013;34(12):1070-3.

15. Beyer R, Kongsgaard M, Hougs Kjaer B, Ohlenschlaeger T, Kjaer M, Magnusson SP. Heavy Slow Resistance Versus Eccentric Training as Treatment for Achilles Tendinopathy: A Randomized Controlled Trial. The American journal of sports medicine. 2015;43(7):1704-11.

16. Heinemeier KM, Skovgaard D, Bayer ML, et al. Uphill running improves rat Achilles tendon tissue mechanical properties and alters gene expression without inducing pathological changes. Journal of applied physiology. 2012;113(5):827-36 .

17. de Oliveira RR, Bezerra MA, de Lira KD, et al. Aerobic physical training restores biomechanical properties of Achilles tendon in rats chemically induced to diabetes mellitus. Journal of diabetes and its complications. 2012;26(3):163-8.

18. Bezerra MA, da Silva Nery C, de Castro Silveira PV, et al. Previous physical exercise slows down the complications from experimental diabetes in the calcaneal tendon. Muscles, ligaments and tendons journal. 2016;6(1):97-103. 
19. de Senna PN, Xavier LL, Bagatini PB, et al. Physical training improves non-spatial memory, locomotor skills and the blood brain barrier in diabetic rats. Brain research. 2015;1618:75-82.

20. de Lacerda Brito ACN, Martins WA, da Silva Queiroz PC, et al. Standardization of a Treadmill Exercise Intensity Protocol in Rats with Diabetes Mellitus. Journal of Exercise Physiology Online. 2019;22(2).

21. Padulo J, Oliva F, Frizziero A, Maffulli NJMLTJ. Basic principles and recommendations in clinical and field science research: 2018 update. 2018;8(3):305-7.

22. de Senna PN, Ilha J, Baptista PP, et al. Effects of physical exercise on spatial memory and astroglial alterations in the hippocampus of diabetic rats. Metabolic brain disease. 2011;26(4):269-79.

23. Carvalho ENd, Carvalho NASd, Ferreira LM. Experimental model of induction of diabetes mellitus in rats. Acta Cirurgica Brasileira. 2003;18(SPE):60-4.

24. Erdal N, Gurgul S, Demirel C, Yildiz A. The effect of insulin therapy on biomechanical deterioration of bone in streptozotocin (STZ)-induced type 1 diabetes mellitus in rats. Diabetes research and clinical practice. 2012;97(3):461-7.

25. Rodrigues B, Figueroa DM, Mostarda CT, Heeren MV, Irigoyen MC, De Angelis K. Maximal exercise test is a useful method for physical capacity and oxygen consumption determination in streptozotocin-diabetic rats. Cardiovascular diabetology. 2007;6:38

26. Silveira ACM, Nery CAdS. Estudo macroscópico e morfométrico do tendão do músculo tibial posterior. Rev bras ortop. 1999;34(8):475-80.

27. Nakagawa S, Cuthill IC. Effect size, confidence interval and statistical significance: a practical guide for biologists. Biol Rev Camb Philos Soc. 2007;82(4):591-605.
28. Jin HY, Lee KA, Park TS. The effect of exercise on the peripheral nerve in streptozotocin (STZ)-induced diabetic rats. Endocrine. 2015;48(3):826-33.

29. Lima-Silva AE, Fernandes TC, De-Oliveira FR, Nakamura FY, Gevaerd MdS. Metabolismo do glicogênio muscular durante o exercício físico: mecanismos de regulação. Revista de Nutrição. 2007.

30. Hargreaves M. Exercise, muscle, and CHO metabolism. Scandinavian journal of medicine \& science in sports. 2015;25 Suppl 4:29-33.

31. Silva RPM, Santos ROd, Junior M, et al. Influence of the use of testosterone associated with physical training on some hematologic and physical parameters in older rats with alloxan-induced diabetes. Archives of endocrinology and metabolism. 2017;61(1):62-9.

32. Coombes B, Tucker K, Hug F, et al. Relationships between cardiovascular disease risk factors and Achilles tendon structural and mechanical properties in people with Type 2 Diabetes. 2019;9(3).

33. Pontieri FM, Bachion MM. Crenças de pacientes diabéticos acerca da terapia nutricional e sua influência na adesão ao tratamento. Ciência \& saúde coletiva. 2010;15:151-60.

34. Green S, Kiely C, O'Connor E, et al. Effects of exercise training and sex on dynamic responses of $\mathrm{O} 2$ uptake in type 2 diabetes. 2020(ja).

35. Snedeker JG, Gautieri A. The role of collagen crosslinks in ageing and diabetes-the good, the bad, and the ugly. Mucles Ligaments Tendons J. 2014;4(3):303.

36. Oliveira RR, Medina de Mattos R, Magalhaes Rebelo L, et al. Experimental Diabetes Alters the Morphology and Nano-Structure of the Achilles Tendon. PloS one. 2017;12(1):e0169513.

37. Boivin GP, Elenes EY, Schultze AK, et al. Biomechanical properties and histology of $\mathrm{db} / \mathrm{db}$ diabetic mouse Achilles tendon. 2014;4(3):280. 